\title{
Enhanced fertility after diagnostic hysterosalpingography using water-soluble contrast media
}

\begin{tabular}{ccc}
\hline Media Ghazi Sedeq * & Aryan Xalis Jawad ** & Shawnm Nasih Dawood * \\
\hline Abstract
\end{tabular}

Background and objective: A possible therapeutic effect of diagnostic tubal patency testing has been debated in the literature for more than half a century. The aim of this study was to evaluate the effect of flushing the fallopian tubes with water-soluble contrast medium during hysterosalpingography on subsequent pregnancy rates and to find out diagnostic findings of hysterosalpingography in infertile patients.

Methods: A prospective study was conducted on 220 women. hysterosalpingography using water-soluble contrast media was done in a private clinic as part of their infertility work up from January 2012 to July 2012 in Erbil city, Kurdistan region, Iraq. The pregnancy rate was determined within six months from the procedure using water soluble contrast media and it was correlated with different hysterosalpingography findings.

Results: The mean age (+SD) of the study population was $30.59(+5)$ years. The clinical pregnancy rate was $23.2 \%$. A statistically significant difference was found between the rate of pregnancy and hysterosalpingography results in which $27.2 \%$ of patients with normal hysterosalpingography and $6.8 \%$ of patients with abnormal hysterosalpingography got pregnant during six-month follow-up period after the procedure $(P=0.004)$.

Conclusion: Flushing of the tubes with water-soluble contrast media during hysterosalpingography increases subsequent pregnancy rates in infertile patients.

Keywords: Hysterosalpingography, Water-soluble contrast media, Pregnancy rate, Infertility

Introduction whether the choice of contrast material used in hysterosalpingography (HSG) influences subsequent reproductive success independent of other therapeutic interventions. ${ }^{1,2}$ HSG is the gold standard and cost effective method of assessing the integrity of the female genital tract. The first HSG performed by Rindfleisch in 1910 when he injected Bismuth solution into the uterine cavity and was considered to be the first interventional radiological procedure. ${ }^{3}$ The main clinical indication for HSG is infertility; either with or without repeated miscarriages. ${ }^{4}$ Uterine abnormalities that can be observed at HSG include congenital anomalies, synechiae, leiomyomas, polyps and adenomyosis. Tubal abnormalities that can be detected with this technique include salpingitis, tubal occlusion, hydrosalpinx, and peritubal adhesions. ${ }^{5,6} \mathrm{~A}$ properly performed HSG provides information on the contour of the uterine cavity and the width of cervical canal. Further injection outlines the cornua, isthmic and ampullary portions of the tubes and evaluates the degree of spillage. ${ }^{7}$ If a properly performed HSG shows the uterine cavity to be normal, it is unlikely that other modalities such as transabdominal, endovaginal, and hysterographic ultrasonography (US) and magnetic resonance (MR) imaging will reveal abnormalities. ${ }^{8,9}$ To our knowledge this research is regarded to be the first to be conducted in our city to determine the effect of HSG on fertility rate. The aim of this study was to (1) find out the rate of

* Department of surgery, college of medicine, Hawler medical university, Erbil, Iraq.

* Department of obstetric and gyenecology, college of medicine, Hawler medical university, Erbil, Iraq. 
http://dx.doi.org/10.15218/zjms.2015.0013

pregnancy after HSG using water-soluble contrast medium (WSCM), (2) find out the types of abnormalities diagnosed by HSG and (3) find out the rate of pregnancy by HSG findings.

\section{Methods}

This study is a prospective review of 220 women referred for HSG as part of their infertility work-up from January 2012 to July 2012 in a private clinic in Erbil city, Kurdistan region, Iraq. The study was approved by the Research Ethics Committee of the College of Medicine in Hawler Medical University. Women were recruited in the study after giving their verbal consent to participate in the study. All the participants were referred by gynecology specialists and had history of either primary or secondary infertility. Women with infertility related to ovarian causes, or related to male partners, were excluded from the study. The preparation for HSG commenced with a detailed history taking emphasizing on the woman's reproductive history aimed at identifying predisposing risk factors associated with infertility. These predisposing risk factors included history of septic abortion, use of intra-uterine contraceptive device, pelvic inflammatory disease (PID), appendicitis, ectopic pregnancy and tubal or other abdomino-pelvic surgery. HSG is an out-patient radiological procedure that involves $X$-rays. The procedure was performed in the first half of the menstrual cycle, after the last day of menstrual flow and before expected day of ovulation. ${ }^{8,9}$ All women received $20 \mathrm{mg}$ intravenous injection of hyoscine butyl bromide 15-20 minute prior to the procedure to minimize discomfort and pain resulting from uterine/ tubal spasm and peritoneal irritation by contrast media. All patients were asked to refrain from unprotected sexual intercourse from the date of her period until after the investigation to be certain there is no risk of pregnancy. The patient was positioned supine on the X-ray table. First plain film was taken to detect any bony abnormality or calcifications and then placed in the lithotomy position, the perineum was cleansed with a povidone-iodine solution and draped with sterile towels. After that a bi-valve speculum was inserted into the vagina and opened to expose the cervix. A special HSG cannula or a pediatric self- retaining catheter was used according to the size of cervix. Then about $10 \mathrm{ml}$ of WSCM (Meglumine compound $76 \%, 370 \mathrm{mg}$ lodine $/ \mathrm{ml}$ ) was injected slowly until the uterine cavity was distended, the tubes filled and contrast seen to spill freely from the distal ends of the tubes. Spot films were taken during the early filling phase to ensure small filling defects were not obliterated by contrast and after complete filling of the tubes to demonstrate free peritoneal spill. Delayed films were taken when early free peritoneal spillage. After the images were taken the catheter and the speculum were removed and the patient given sanitary towel to absorb the contrast agent. Results of all the HSGs were evaluated as being normal or abnormal. A normal HSG shows a healthy, normally shaped uterus and unblocked fallopian tubes which permits egress of free inverted comma shaped contrast spillage into the peritoneal cavity bilaterally. Abnormal tubal and uterine findings as uni- or bilateral tubal blockage with or without hydrosalpinx and uterine anomalies were determined and frequency of different abnormalities was detected. All women were followed-up for six months after the procedure and pregnancy was diagnosed by positive hormonal test and seeing gestational sac within the uterine cavity by pelvic ultrasound examination. The rate of pregnancy was then calculated and correlated with certain variables. HSG was well-tolerated by the patients though we found some difficulties in certain cases like inadequate distension of the uterus due to cervical reflux or narrow external cervical os. This problem was solved by using adequate-sized cannulas. Tubal spasm which can give rise to a false positive diagnosis of cornual occlusion was 
http://dx.doi.org/10.15218/zjms.2015.0013

avoided by slow injection of the contrast medium and giving antispasmodics half hour prior to the procedure. Routine use of antibiotics is controversial ${ }^{10}$ but prophylactic antibiotics were not used in our work.

\section{Statistical Analysis}

Statistical package for the social sciences (version 21.0) was used for data entry and analysis. Both descriptive and analytic approaches were used; descriptive to determine the frequencies, means, and standard deviation; and analytic by using Chi-square test to find out associations. A $P$ value $\leq 0.05$ was regarded statistically significant.

\section{Results}

Of 220 women, 101 (46\%) had primary

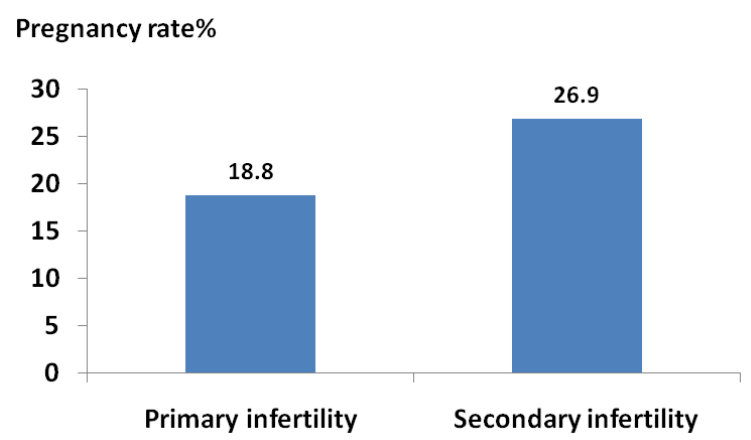

Figure 1: Pregnancy rate in relation to type of infertility. infertility and 119 (54\%) had secondary infertility. The age range of the infertile women was 20-45 years with mean $( \pm S D)$ of $30.59 \pm 5$ years. The study revealed an overall pregnancy rate of $23.2 \%$ with a statistically non-significant difference between those with primary or secondary infertility, $(P=0.157)$ as shown in Figure 1. From the total of 220 HSG; 176 (80\%) were normal and $44(20 \%)$ were abnormal showing different pathologies (Figure 2). A statistically significant difference was found between the rate of pregnancy and HSG results where $27.2 \%$ of patients with normal HSG and $6.8 \%$ of patients with abnormal HSG got pregnant during six-month follow-up period after the procedure, $(P=0.004)$ as shown in Figure 3.

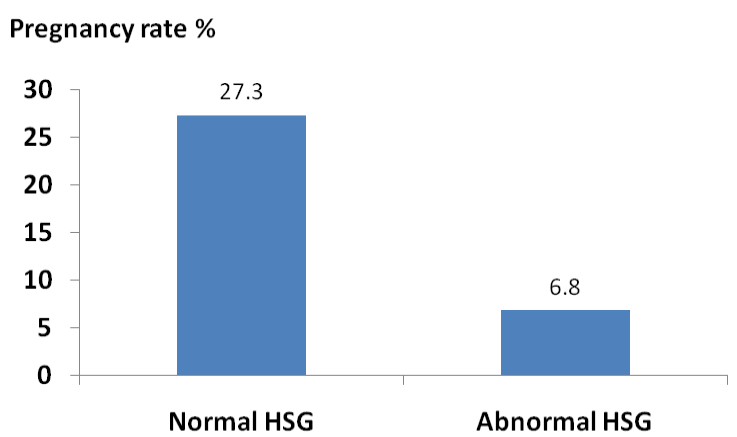

Figure 3: Rate of pregnancy in relation to HSG results
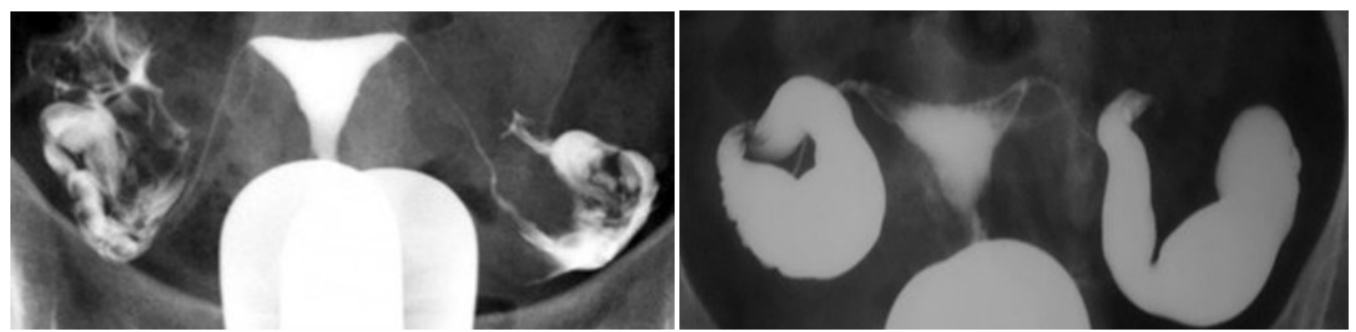

Figure 2: A. Normal hysterosalpingogram. A smooth triangular uterine cavity and spill from both tubes. B. Bilateral blocked tubes. HSG showing a normal uterus and blocked tubes. No "spill" of dye is seen at the ends of the tubes. Both tubes are slightly dilated and fluid filled (hydrosalpinx). 
There was no statistically significant difference regarding the use of intrauterine contraception, history of previous abdominal operation and pelvic inflammatory disease and the pregnancy rate (Table1). There was no statistically significant association between different abnormal HSG findings and rate of pregnancy as two patients with right side patent hydrosalpinx and only one patient with bilateral patent hydrosalpinx got pregnant (Table 2).

Table 1: Association between certain variables with pregnancy rate.

\begin{tabular}{llllllll}
\hline Variables & \multicolumn{2}{l}{ Pregnant } & \multicolumn{2}{l}{ Not pregnant } & \multicolumn{2}{l}{ Total } & p \\
& No. & $\%$ & No. & $\%$ & No. & $\%$ & \\
\hline $\begin{array}{l}\text { Use of contraception } \\
\text { Yes }\end{array}$ & 9 & 26.5 & 25 & 73.5 & 34 & 100 & \\
No & 42 & 22.6 & 144 & 77.4 & 186 & 100 & 0.62 \\
PID & & & & & & & \\
Yes & 4 & 40 & 6 & 60 & 10 & 100 & \\
No & 47 & 22.4 & 163 & 77.6 & 210 & 100 & 0.246 \\
Operation & & & & & & & \\
Yes & 18 & 21.2 & 67 & 78.8 & 85 & 100 & \\
No & 33 & 24.4 & 102 & 75.6 & 135 & 100 & 0.576 \\
\hline
\end{tabular}

Table 2: Association between abnormal HSG findings and rate of pregnancy.

\begin{tabular}{|c|c|c|c|c|c|c|c|}
\hline \multirow[t]{2}{*}{ HSG abnormal findings } & \multicolumn{2}{|c|}{ Pregnant } & \multicolumn{2}{|c|}{ Not pregnant } & \multicolumn{2}{|c|}{ Total $=44$} & \multirow[t]{2}{*}{ p } \\
\hline & No. & $\%$ & No. & $\%$ & No. & $\%$ & \\
\hline Obstructed right tube & 0 & 0 & 4 & 100 & 4 & 100 & \multirow{15}{*}{$0.116^{*}$} \\
\hline Obstructed left tube & 0 & 0 & 4 & 100 & 4 & 100 & \\
\hline Obstructed right tube with hydrosalpinx & 0 & 0 & 3 & 100 & 3 & 100 & \\
\hline Bicornuate uterus & 0 & 0 & 10 & 100 & 10 & 100 & \\
\hline Unicornuate uterus & 0 & 0 & 2 & 100 & 2 & 100 & \\
\hline Patent normal tubes but irregular uterus & 0 & 0 & 1 & 100 & 1 & 100 & \\
\hline Hydrosalpinx of right tube but patent & 2 & 40 & 3 & 60 & 5 & 100 & \\
\hline Hydrosalpinx left tube but patent & 0 & 0 & 1 & 100 & 1 & 100 & \\
\hline Obstructed both tubes & 0 & 0 & 5 & 100 & 5 & 100 & \\
\hline Right tube absent (removed) & 0 & 0 & 1 & 100 & 1 & 100 & \\
\hline Left tube removed ( absent) & 0 & 0 & 4 & 100 & 4 & 100 & \\
\hline Small uterus obstructed tubes & 0 & 0 & 1 & 100 & 1 & 100 & \\
\hline $\begin{array}{l}\text { Bilateral hydrosalpinx and bilateral ob- } \\
\text { struction }\end{array}$ & 0 & 0 & 1 & 100 & 1 & 100 & \\
\hline Bilateral hydrosalpinx but patent tubes & 1 & 100 & 0 & 0 & 1 & 100 & \\
\hline Bicornuate didelphys & 0 & 0 & 1 & 100 & 1 & 100 & \\
\hline
\end{tabular}

${ }^{*}$ Fisher Exact test 


\section{Discussion}

It is a known fact that hysterosapingography has both therapeutic and diagnostic values. Following HSG, certain minor/mild uterine adhesions and partial tubal occlusion are lysed and a hitherto infertile woman have conceived months after HSG without any other gynaecological intervention. ${ }^{2,11-14}$ Our study revealed increased pregnancy rate of the infertile women within six months after HSG using WSCM in which the overall pregnancy rate was $23.2 \%$. Several early studies with sample size and methodologic limitations and a meta-analysis of their results indicated that this interpretation is possible. ${ }^{13-17}$ The likelihood of pregnancy was not greater in women with primary infertility than that in women with secondary infertility; this is in agreement with Spring et al. ${ }^{13}$ The detection rate for pathologies at HSG was low $(20 \%)$, which is in agreement with Schankath et $\mathrm{al}^{17}$ that had $21 \%$ rate of HSG pathologies. The study showed $63 \%$ of HSG abnormalities to be tubal in origin. This agrees with other studies that state Fallopian tubal factors are responsible for approximately one third of female infertility ${ }^{5,18}$. From the cases that had abnormal HSG and got pregnant all had tubal abnormalities in the form of patent hydrosalpinx whereas none of patients with uterine malformations got pregnant within the six-month period after the procedure.

\section{Conclusion}

Flushing of the tubes with water-soluble contrast media during HSG increases subsequent pregnancy rates in infertility patients. The overall pregnancy rate was $23.2 \%$ with a statistically non-significant difference between those with primary or secondary infertility.

\section{Conflicts of interest}

The author reports no conflicts of interest.

Acknowledgment: The authors would like to thank professor Namir Ghanim Al-Tawil
(FICMS/CM) and Dr. Samir Othman (PhD/CM) from Community Medicine department/College of Medicine/Hawler medical university for their valuable help in statistical analysis.

\section{References}

1. Soules MR, Spadoni LR. Oil versus aqueous media for hysterosalpingography: a continuing debate based on many opinions and few facts. Fertil Steril 1982; 38:1-11.

2. Watson A, Vandekerckhove P, Lilford R, Vail A, Brosens I, Hughes E. A meta-analysis of the therapeutic role of oil soluble contrast media at hysterosalpingography: a surprising result? Fertil Steril 1994; 61:470-7.

3. Katz VL. Diagnostic procedures: Imaging, endometrial sampling, endoscopy: indications and contraindications, complications. In: Katz VL, Lentz GM, Lobo RA, Gershenson DM, eds. Comprehensive Gynecology. 5th ed. Philadelphia, Pa: Mosby Elsevier; 2007.

4. Simpson WL, Beitia LG, Mester J. Hysterosalpingography: a re-emerging study. RadioGraphics 2006; 26(2):419-31.

5. Miller J, Weinberg R, Nancy L. The pattern of infertility diagnoses in women of advanced reproductive age. Am J Obstet Gynecol.1999; 181 (4):952-7.

6. Eng CW, Tang PH, Ong CL Hysterosalpingography: current applications. Singapore Med J 2007; 48(4):368-73.

7. Socolov D, Boian I, Boiculese L, Tamba B, Socolov R. Comparison of the pain experienced by infertile women undergoing hysterosalpingo contrast sonography or radiographic hysterosalpingography. Int J Gynaecol Obstet 2010; 111(3):256-9.

8. Baramki TA. Hysterosalpingography. Fertil Steril 2005; 83(6):1595-606.

9. Krysiewicz S. Infertility in women: diagnostic evaluation with hysterosalpingography and other 2 imaging techniques. Am J Roentgenol 1992; 159(2): 253-61.

10. Sutton D. Hysterosalpingography. In: Sutton D (editor). Textbook of radiology and imaging: 6th ed. New York: Churchill Livingstone, 2003. P. 1085-6.

11. Rasmussen $F$, Lindequist $S$, Larsen $C$, Justesen $P$. Therapeutic effects of hysterosalpingography: oil- versus water-soluble contrast media-a randomized prospective study. Radiology 1991; 179:75-8.

12. Verhoeve HR, Coppus SF, Steures $P$, Bourdrez $P$. The capacity of hysterosalpingography and laparoscopy to predict natural conception. Hum Reprod 2011; 26(1):134-42

13. Spring DB, Barkan HE, Pruyn SC. Potential therapeutic effects of contrast materials in hysterosalpingography: a prospective randomized 
clinical trial. Kaiser Permanente Infertility Work Group. Radiology 2000; 214(1):53-7.

14.Barqawi R, Bani-Irshaid I. The effects of Contrast Media in Patients Undergoing Salpingography on Pregnancy Rates. JBMS 2007; 19(4)133-6.

15. Court KA, Dare AJ, Weston-Webb M, Hadden WE, Sim RG, Johnson NP. Establishment of lipiodol as a fertility treatment - prospective study of the complete innovative treatment data set. Aust N Z J Obstet Gynaecol 2014; 54(1):13-9.

16. David B. Spring, Howard E. Barkan. Enhanced Fertility After Diagnostic Hysterosalpingography May Be a Myth. AJR 2004; 183:1728.

17. Schankath AC, Fasching N, Urech-Ruh C, Hohl MK, Kubik-Huch RA. Hysterosalpingography in the workup of female infertility: indications, technique and diagnostic findings. Insights Imaging 2012; 3(5):475-83.

18. Mallarini G, Saba L. Role and application of hysterosalpingography and Fallopian tube recanalization. Minerva Ginecol 2010; 62(6): 541-9. 